\title{
Opioid Misuse Among Sexual and Gender Minorities in Texas: The Most Vulnerable Populations
}

\begin{abstract}
Keywords: Prescription opioids; Adolescents; LGBT; HIV risk; Harm reduction

Abstract

Background: Increasing opioid-related mortality is especially concerning in Sexual and Gender Minority (SGM) persons who are more likely than cis-gender heterosexual persons to have substance use disorders. These analyses identified variables associated with opioid (heroin and prescription pain medication) use by SGM Texans.

Method: Analyses were conducted on 1273 SGM Texans who completed an online cross-sectional survey. Variables including demographic characteristics, substance use, and notable life experiences. Variables found to be significant in bivariate analyses were entered into logistic regression models.
\end{abstract}

Results: 128 (10.0\%) of participants reported opioid use within the 12 months prior. Those who reported opioid use were more likely to use another substance or engage in hazardous drinking. Those who reported injection drug use and having a non-monosexual private identity were more likely to report opioid use. Although age was not found to be a significant predictor of opioid use, those under 19 year of age accounted for $57.0 \%$ of those who reported opioid use.

Discussion: Opioid use is a concern among SGM persons, especially those who have a non-monosexual identity. Organizations serving SGM persons provide services to youth should consider implementing a screening, intervention, treatment, and harm reductionfor opioids, alcohol, and other substances.

\section{Introduction}

An estimated 11.4 million people in the United States (U.S.) over the age of 12 years (4.2\%) have misused opioids, and 2.1 million met criteria for an opioid use disorder [1]. In 2017, the time of the data collection in the results presented in this article, $67.8 \%$ of all drug overdose related deaths were due to opioid overdoses, increasing significantly from 2016 by $12.0 \%$ [2]. National data shows a decline in opioid related overdose deaths from 2017 to 2018 of $2 \%$ [3]. Unfortunately, this decline was short-lived. Opioid overdoses are again increasing at alarming rates as the U.S. population deals with stressors associated with coronavirus disease 2019 (COVID-19) [4-6].

Although opioid overdose deaths are highest in the eastern U.S. [7], an increasing number of deaths in other densely-populated states is cause for alarm. For example, in Texas rates of opioid overdose deaths increased from $5.1 \%$ in 2013 to $14.9 \%$ in 2017 , a $9.8 \%$ increase [2]. While this increase is lower than the national increase, incidence rates are still high. There is some concern that these rates could be underestimating the true rate of opioid overdose deaths in the state. Only 15 of 254 counties in Texas have medical examiners that can perform autopsies and verify a death was opioid related [8]. Thus, it

\section{Journal of}

\section{Addiction \& Prevention}

\author{
Di Paola A, McCurdy SA, Schick V and Wilkerson JM* \\ Division of Health Promotion and Behavioral Sciences, School of \\ Public Health, USA

\section{*Address for Correspondence} \\ Wilkerson JM, Center for Health Promotion and Prevention Research, \\ Division of Health Promotion and Behavioral Sciences, School of Public \\ Health, The University of Texas Health Science Center at Houston 7000 \\ Fannin, Suite 2620, Houston, TX 77030, USA, Tel: 713.500.9974, Fax: \\ 713.500.9750; E-mail: Johnny.M.Wilkerson@uth.tmc.edu \\ Submission: 22 December, 2020 \\ Accepted: 28 January, 2021 \\ Published: 30 January, 2021 \\ Copyright: @ 2021 Di Paola A. This is an open access article distributed \\ under the Creative Commons Attribution License, which permits \\ unrestricted use, distribution, and reproduction in any medium, provided \\ the original work is properly cited.
}

is plausible that the rate of opioid overdose is higher in Texas, and perhaps in similar states with limited numbers of medical examiners.

Although opioid overdoses have increased in almost all demographic characteristics [2], persons most likely to report misusing or meet Diagnostic and Statistical Manual of Mental Disorders (DSM-5) criteria for an opioid use disorder include those who are uninsured, unemployed, or have lower family income levels [9]. Young adults between the ages of 18 and 25 are effected by the epidemic more than older adults. It is estimated that $7.3 \%$ of young adults misuse opioids and $1.3 \%$ meet criteria for an opioid use disorder [1].

In general, Sexual and Gender Minority (SGM) persons ages 18 and older are more than twice as likely to misuse substances than their cisgender, heterosexual peers (39.1\% vs. 17.1\%), including misuse of prescription opioids (10.4\% vs. $4.5 \%)$ [10]. They are also more likely than their peers to meet DSM- 5 criteria for a substance use disorder, including an opioid use disorder [11-13]. State-level rates of opioid misuse within the SGM community are limited, and factors associated with opioid use by SGM persons remain largely unexplored.

High rates of prescription opioid misuse have been linked to the initiation of heroin use [14-16], injection drug use [17], HIV and hepatitis C transmission [18-20], and an increased risk of opioid overdose $[18,21]$. Given the already elevated rate of HIV among men who have sex with men and transgender women [22,23], it is important to identify SGM persons most at risk for experiencing opioid-related adverse health eventsto introduce secondary and indicated prevention and treatment interventions.

The minority stress theory proposes that various environmental factors which are less supportive of an individual's identity leads to stress producing circumstances including rejection and internalization contribute to negative mental health and behavioral health outcomes including substance use [24-26]. The individual identities and factors that are included in the theory include: sexual identity, gender, and race/ethnicity, general stressors, discrimination, and prejudice based 
Citation: Di Paola A, McCurdy SA, Schick V, Wilkerson JM. Opioid Misuse Among Sexual and Gender Minorities in Texas: The Most Vulnerable Populations. J Addiction Prevention. 2021;9(1): 7.

ISSN: $2330-2178$

Table 1: Demographic characteristics and bivariate analyses results.

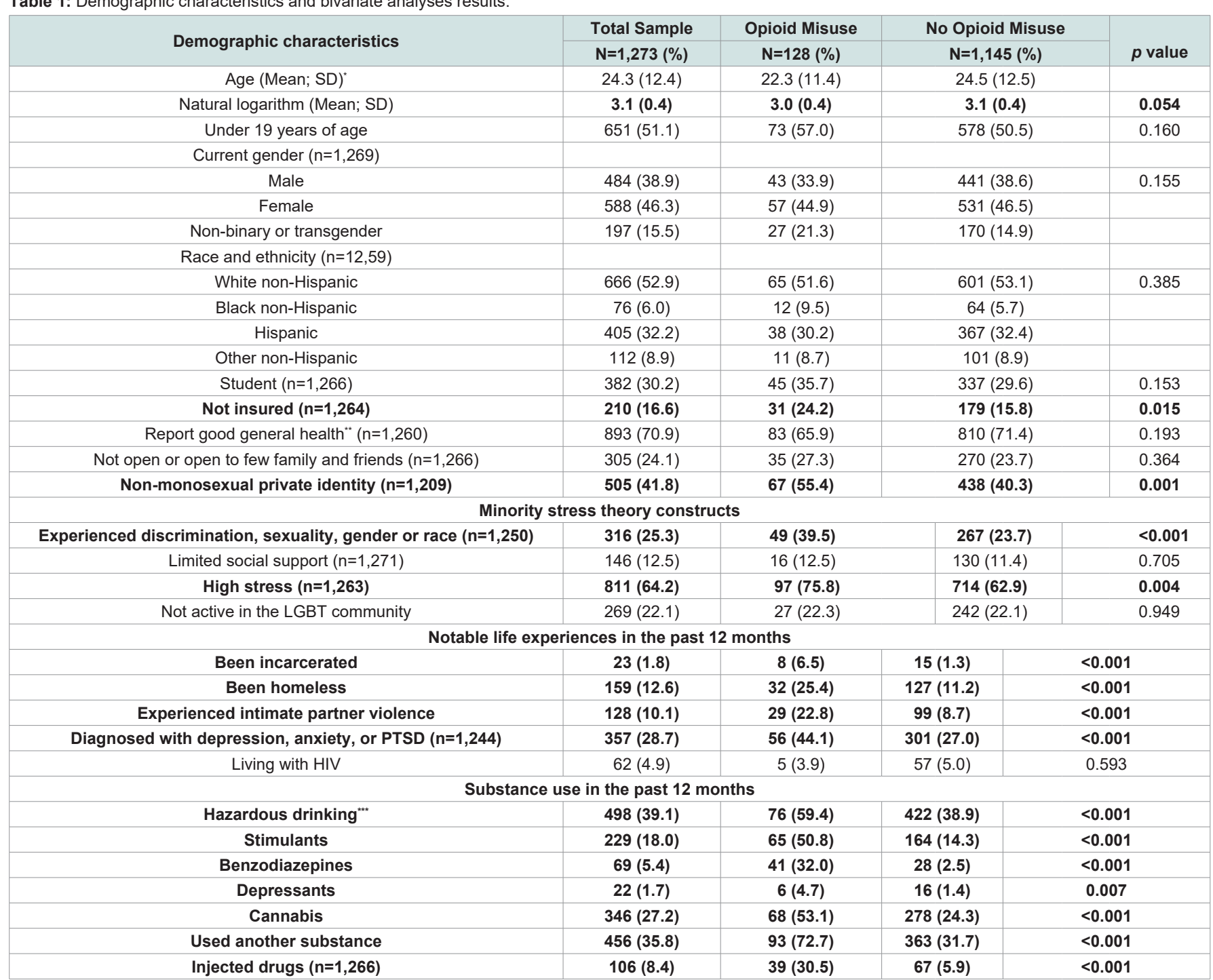

"bivariate analysis not conducted on age variable due to skewness and kurtosis, the transformed natural logarithm was used to correct this.

** quality of life item from CDC HRQOL-4

"*thazardous drinking per AUDIT-C criteria

on the individual's identity [24-26]. Previous research has shown that SGM persons who experience discrimination have higher rates of problematic alcohol consumption $[27,28]$, substance use disorders [29-31], and injection drug use [27], than those that do not experience discrimination. In comparison to cisgender heterosexual persons, SGM persons are also more likely to report life experiences such as homelessness and intimate partner violence [32-36]. The purpose of this article is to explore the prevalence rate of opioid misuse, type of opioids used, social factors, and theory-based constructs associated with opioid misuse among SGM persons.

\section{Methods}

\section{Study design}

Using social media banner ads, participants were recruited in collaboration with SGM-serving community centers to complete a statewide online cross-sectional survey, Tell Us, Texas. Social media banner ads targeted toward persons age $13+$ who indicated in their profile that they were a sexual or gender minority living in Texas or were interested in at least one SGM-related topic (e.g., Gay Pride, LGBT bars, or LGBT community centers). SGM-serving community centers referred persons to the survey link. Between March 2016 and January 2017, participants completed the survey $(\mathrm{N}=1273)$ in English or Spanish. To be eligible for the study, participants must have been living in Texas, been 13 years of age or older, and identified as a sexual or gender minority. To verify participants lived in Texas and remove invalid entries, we adapted a previously published protocol to include a Texas zip code [37]. Persons that did not have a Texas zip code were excluded from the analyses. Participants completed an online consent form prior to viewing the survey, which took approximately 20-minutes to complete. Upon completion, the first 1,000 participants received a $\$ 5$ e-gift card. All study protocols were reviewed and approved by the researcher's institutional review board.

\section{Measures}


Researchers at The University of Texas Health Science Center at Houston and staff at a local SGM-serving community center developed the survey. It included questions about demographic characteristics, HIV sexual risk behaviors, minority stress theorybased constructs, physical and mental health diagnoses, substance use, alcohol consumption, and various life experiences. This analysis focused on the association between opioid misuse and individual demographic characteristics, minority stress theory-based constructs, notable life experiences, and co-occurring substance and alcohol use.

Demographic characteristics included gender, age, race/ethnicity, student status, insurance status, and perceived quality of life. The majority of these variables were converted to categorical variables for these analyses, with the exception of age. Participants were asked to indicate their sex at birth and current gender. Using this twostep method [38], we categorized participants as cisgender men (assigned male at birth and identified as a men), cisgender women (assigned female at birth and identified as awomen), or non-binary/ transgender persons (identified as a different gender than assigned at birth). Participants' responses" to race and ethnicity items were used to categorize participants as Hispanic/Latinx, White non-Hispanic, Black non-Hispanic, or other non-Hispanic. We created dichotomous (yes/no) variables using participants' responses" to questions about whether or not they were a student or had health insurance. We also created a dichotomous variable using participants" responses to one item from the Center for Disease Control and Prevention Health Days core questions (CDC HRQOL-4), which asked, "Would you say that in general your health is" with responses of a five-point Likertscale from "excellent" to "poor". Those who responded being in "good", "very good", or "excellent" health were categorized as being in good general health [39]. Due to the positive skewness and kurtosis of age, the natural logarithm was included in analyses as a continuous variable.

Participants were asked to indicate their sexual identity. To compare health disparities between participantsidentifying as nonmonosexual or monosexual [40,41], we categorized participants identifying as heterosexual or homosexual as monosexual, and categorized participants identifying as another sexual orientation, including bisexual, pansexual, and queer, as non-monosexual. Participants were asked how open to others they were about their sexual orientation. Response options were on a 5-point Likert scale ranging from not at all out to out to most or all people [42]. A dichotomous variables was created to compare participants who were out to few family and friends ( 1 and 2 ) to those who were out to half or more family and friends (3 to 5 ).

Minority stress theory-based constructs were also explored in these analyses. Participants were asked if they had experienced discrimination in the past month to completing the survey based on their sexuality, gender, or race/ethnicity [43]. Response options were on four-point Likert-scale ranging from "never" to "often." A dichotomous variable was created to compare participants reportingany recent discrimination to those with no ("never") recent discrimination. A single-item social support question was used to determine the individual's level of social support. Those reporting have little or no social support were compared to those who reported higher levels of social support [44]. On a 6-point Likert scale, participants were asked about their current level of stress from "Not at all" to "Very much" [45]. Using a cutoff value of 3, we created a dichotomous variable to compare participants with higher orlower levels of stress. Participants were also asked items regarding their level of involvement in the Lesbian, Gay, Bisexual, and Transgender (LGBT) community.We created a dichotomous variable to compare those who were to those who were not active in the LGBT community.

In order to identify other factors associated with opioid use participants were asked if they had experienced a verity of notable life events. Participants were asked to indicate if and when they experienced incarceration, homelessness, or intimate partner violence. They were also asked to indicate if and when they had received a diagnoses for psychological distress (depression, anxiety or panic disorder, or Post-Traumatic Stress Disorder [PTSD]) and if they were diagnosed with HIV. Response options for all items included never, within the past 30 days, within the past 12 months, or more than a year ago. Participants' responses were dichotomized to compare those who did or did not report experiencing each life event within the 12 months prior to completing the survey.

Alcohol and substance use behaviors were collected for the 12 months prior to completing the survey. The three item Alcohol Use Disorder Identification Test (AUDIT-C) was used to determine if participants met criteria for hazardous drinking within the 12 months prior to completing the survey $[46,47]$. Those with a score of three or above for women or four or above for men where coded as being a hazardous drinker based on gender assignment at birth. Participants were also asked if they misused various substances within the 12 months prior to completing the survey. These substances were grouped by drug category: stimulants (e.g. ecstasy, ADHD medications, methamphetamine, and cocaine), benzodiazepines (e.g. Valium, and Ativan), depressants (e.g. GHB and rohypnol), and cannabis taken illicitly or not as medically prescribed. Due to the high prevalence of polysubstance use, a collective substance misuse variable was created to better understand the social determinates of opioid misuse in this population. Participants were also asked if they had injected any of the substances. A dichotomous variable was created to compare participants who reported a history of injection drug use versus those who did not.

\section{Outcome variable}

Similar to the substance misuse categories, participants were asked if they had used various substances illicitly or not as medically prescribed within the 12 months prior to completing the survey. Those who reported misuse of any of the individual opioids were coded as having misused opioids in the 12 months prior to completing the survey. Individual items including heroin, prescription pain medication (e.g. Vicodin and Dilaudid), cough syrup with codeine, buprenorphine, or methadone.

\section{Analyses}

Chi-squared, Fisher's exact, or t-tests were used to determine if there were differences at a bivariate level for the various participant demographic characteristics, minority stress theory-based constructs, notable life experiences, hazardous drinking, and substance misuse among those who did or did not report opioid misuse within the 12 months prior to completing the survey. Those found statistically 
Table 2: Logistic regression analysis results of opioid misuse.

\begin{tabular}{|c|c|c|c|}
\hline \multirow{2}{*}{\begin{tabular}{|l|} 
Variable \\
Natural logarithm age \\
\end{tabular}} & \multirow{2}{*}{$\begin{array}{l}\text { Adjusted Odds Ratio } \\
1.67\end{array}$} & \multicolumn{2}{|c|}{$95 \%$ Confidence Interval } \\
\hline & & 0.94 & 2.95 \\
\hline Not insured & 1.30 & 0.78 & 2.18 \\
\hline Non-monosexual private identity & 1.73 & 1.11 & 2.69 \\
\hline Experienced discrimination & 1.06 & 0.65 & 1.74 \\
\hline High stress & 1.27 & 0.77 & 2.08 \\
\hline Been incarcerated & 2.28 & 0.73 & 7.13 \\
\hline Been homeless & 0.90 & 0.50 & 1.64 \\
\hline Experienced Intimate Partner Violence & 1.13 & 0.62 & 2.06 \\
\hline Diagnosed with depression, anxiety, or PTSD & 1.03 & 0.64 & 1.68 \\
\hline Hazardous drinking & 2.15 & 1.39 & 3.32 \\
\hline Used another substance & 3.76 & 2.35 & 6.01 \\
\hline Injected drugs & 3.19 & 1.70 & 6.02 \\
\hline
\end{tabular}

significant at a $\mathrm{p}<0.10$ level were entered into a logistic regression model $[48,49]$. Due to the high rate of polysubstance use, the combination substance misuse variable was used in the regression models and not the individual substances. Variables with a $\mathrm{p}<0.05$ were considered to be statistically significant in the logistic regression. All analyses were conducted in STATA version 14 [50].

\section{Results}

This was a young sample averaging 24.3 years of age (range 13 to 67 years), $51.1 \%$ of the sample was under the age of 19 years. Among participants, $46.3 \%$ reported being a cisgender female, $38.9 \%$ reported being a cisgender male, and $15.5 \%$ reported being a nonbinary or transgender person. The sample was ethnically and racially diverse; $32.2 \%$ identified as Hispanic/Latinx and 6.0\% identified as non-Hispanic Black. More than half (58.2\%) reported having a monosexual private identity, the majority of whom identified as gay or lesbian (91.3\%). Among the $41.8 \%$ reported having a nonmonosexual private identity $63.5 \%$ identified as bisexual, $17.4 \%$ as pansexual, and $19.0 \%$ as queer. Nearly a third of the sample (30.2\%) identified as students, and $16.6 \%$ reported not having health insurance. A quarter (25.3\%) of the participants reported having experienced sexual, gender, or race-based discrimination, and 28.7\% reported being diagnosed with either depression, anxiety, or PTSD within the 12 months prior to completing the survey.

Of the full sample, $10.0 \%(\mathrm{n}=128)$ reported having misused an opioid. The most commonly misused opioids were prescription pain medications $(79.7 \%, \mathrm{n}=102)$, cough syrup with codeine $(25.6 \%$, $\mathrm{n}=20)$, and heroin $(12.3 \%, \mathrm{n}=17)$. Those accounting for the majority of the opioids misuse were those under the age of 19 years $(57.0 \%$ of the opioid users).

\section{Bivariate level analysis}

Results of bivariate analyses are summarized in (Table 1). Those who reported opioid misuse were younger than those who did not report misusing opioids in the 12 months prior to completing the survey $(\mathrm{p}=0.054)$ and uninsured $(\mathrm{p}=0.015)$. Opioid misuse was more common among participants out to most or all people they knew ( $\mathrm{p}=0.048$ ), and among those who had a non-monosexual private identity $(\mathrm{p}=0.001)$. Those who reported opioid misuse also reported experiencing discrimination $(\mathrm{p}<0.001)$ or high levels of stress $(\mathrm{p}=0.004)$. Participants with a history of incarceration, homelessness, intimate partner violence, or a recent mental health diagnoses (all $\mathrm{p}<0.001$ ) were also likely to report opioid misuse. Opioid misuse was also more common among participants reporting other substance use $(p<0.001$ for all categories and the combined variable except depressants $\mathrm{p}=007)$, hazardous drinking, $(\mathrm{p}<0.001)$, and for those who engaged in injection drug use $(\mathrm{p}<0.001)$.

\section{Logistic regression}

Variables found to be statistically significant $(p<0.10)$ at the bivariate level were entered into a logistic regression model to obtain adjusted odds ratios for factors most strongly associated with opioid misuse (Table 2). Participants with a non-monosexual identity (adjusted Odds Ratio [aOR] 1.73; 95\% Confidence Interval [CI]: $1.11,2.69)$ were more likely misuse opioids. Participants reporting using another substance (aOR 3.76; 95\% CI: 2.35, 6.01), engaging in hazardous drinking (aOR 2.147; 95\% CI: 1.39, 3.32;), or engaging in injection drug use (aOR 3.19; 95\% CI: 1.70, 6.02) were also more likely to misuse opioids.

\section{Discussion}

SGM Texans misused opioids at rates similar to those reported else where using data from a national sample (10.0\%) [10]. What is perhaps unique to Texas is the high off-label use of prescription pain medication (79.7\% of the opioid misused), which is associated with the initiation of heroin use and injection drug use [14,15,17]. It is plausible that Texas will see an increasing number of SGM persons using and injecting heroin. Surveillance efforts should include monitoring of SGM substance use. These analyses also show that of those who misused opioids in the 12 months prior to completing the survey had an increased rate of hazardous drinking, other substance misuse, and engage in injection drug use behavior. Therefore, a more effective screening and referral to substance use treatment is needed.

SGM persons over the age of 18 have higher rates of opioid use then their cisgender peers [10]. What is notable from our sample is the high proportion of opioid using adolescents. Of the 128 opioid users in our sample, 57\% were under 19 years of age. Furthermore, within our sample, $11.2 \%$ of those between 13 and 18 years old reported opioid misuse, far exceeding the national estimate of $3.1 \%$ for adolescents between the ages of 12 and 17 years [1]. It is imperative that SGM-serving youth organizations, pediatricians and adolescent physicians screen SGM patients for substance misuse.

Non-monosexual participants were more likely to report opioid misuse than monosexual participants. There is a growing body of literature suggesting that among SGM persons, those who express 
attraction to individuals of more than one gender are more likely to experience negative health outcomes [32,35,36]. An application of minority stress theory offers a plausible explanation for this difference $[24,25]$. It is plausible that non-monosexual persons experience more discrimination due to their sexual minority status than monosexual persons, including persons identifying as gay or lesbian. These discriminatory experiences could result in more psychological distress and hostility and distrust of monosexual persons outside of and within the SGM community [51]. This psychological distress could intern contribute to greater social isolation by non-monosexual persons, missing potential psychological benefits of social support experienced by persons with monosexual identities [41], an important moderator of the relationship between minority stress and health outcomes [52]. While our findings suggest all SGM persons should be screened for a substance use disorder and linked to culturally competent social services, it is important to ask nuanced questions about a client's sexual identity and behavior so that non-monosexual individuals can be appropriately directed to providers who are considerate of the unique challenges that they may encounter.

Although not found to be significant in the logistic regression, some demographic characteristics and notable life eventsfoundto be significant at the bivariate level provide additional guidance on how to tailor interventionsand increase access to culturally competent healthcare for opioid-using SGM persons. Compared to participants not using opioids, more participants reporting opioid misuse lacked health insurance; decreasing access to routine medical care, emergency services, and substance use detoxification, treatment, and recovery services [53-58]. This lack of health insurance is more concerning in a non-Medicaid expansion state, like Texas, where uninsured persons might face additional financial barriers to accessing healthcare. Future researchers might want to assess the cultural competency to serve substance-using SGM persons of staff at federally qualified health centers and other community-based clinics to determine if additional training and support is needed to increase the willingness of substance-using SGM persons to access their healthcare services.

Those who reported opioid misuse were also more likely to report recent discrimination based on sexuality, gender, or race. We did not ask about discrimination based on recent substance use. However, SGM persons sometimes delay accessing healthcare to avoid potential discrimination by a healthcare provider [54,55], as do persons with a substance use disorder [59,60]. This is an important area for future research. By identifying sources of discriminationespecially discrimination that might impact accessing health services-researchers, practitioners, and policy makers might identify new collaborative efforts to develop and evaluate structural-level interventions that reduce stigmatization based on sexuality, gender, race/ethnicity, and substance use.

Within this sample $6.5 \%$ of persons who reported misusing opioids were incarcerated compared to $1.3 \%$ of those who did not use opioids. Given the small number of previously incarcerated persons in the sample, it is likely that a larger sample would have found a significant difference in the logistic regression. Similarly, this regression was not powered to determine the effect of those that have experienced homelessness, discrimination, intimate partner violence, and having been diagnosed with a mood or anxiety disorder among those who reported opioid misuse. Yet these are important experiences that should be considered when screening for opioid and other substance misuse, as well as when developing a treatment plan for each individual.

Concerning are the changes in substance use related overdose during the COVID-19 pandemic. During the early months of COVID-19 pandemic, March to May 2020, the largest monthly increase in substance use related deaths since 2015 was record [6]. Between June 2019 and May 2020, nationally there was a 20\% increase substance use related overdoses, primarily driven by a $38.4 \%$ increase in synthetic opioid related overdose deaths [6]. Alarmingly the increase in synthetic opioid related overdose deaths in Texas, as well as 16 other states, increased over $50 \%$ in that timeframe [6]. Given SGM persons have higher rates of opioid use than their cisgendered peers, there is an urgent need for research, prevention, and treatment services in this community.

Although we were able to obtain a large sample of only SGM persons across Texas, including a subsample of persons currently engaging in substance misuse, there are some limitations to this study. This was a cross-sectional study, therefore changes over time and causality cannot be determined. However, participants were recruited using social media ads, and considering that the majority of the U.S. population use social media these results may able to be generalizable to many SGM Texans, and possibly nationally [61]. A second limitation of our study was the age of the sample. While recruiting a mostly young sample resulted in persons most likely to misuse opioids, the lack of older participants prohibited a comparisons of opioid use across age cohorts. This study is the use of brief self-report measures to reduce the burden on participants completing the survey. The use of more extensive psychosocial and cultural measures would likely add to our understanding of the effect of discrimination and notable life experiences on SGM persons who misuse opioids.

\section{Conclusion}

These results support the need for integrated and tailored screening, harm reduction, and treatment interventions with SGM culturally competent care providers or community-based organizations, especially among adolescence. Some of the possible interventions include harm reduction education and naloxone distribution for those at risk for overdose. In addition, pediatricians treating large numbers of adolescents might wish to implement routine screening, referral, and linkage for substance use disorders. Our findings also support the need for further integration of mental health and trauma related services with substance use treatment and recovery support services, and increased access to medication assisted treatment, telehealth services, recovery coaches, recovery residences, and peer-based support groups to provide more holistic care solutions.

\section{References}

1. Substance Abuse and Mental Health Services Administration, Key substance use and mental health indicators in the United States: Results from the 2017 National Survey on Drug Use and Health, in HHS Publication No. SMA 18-5068, NSDUH Series H-53. 2018: Rockville, MD: Center for Behavioral Health Statistics and Quality, Substance Abuse and Mental Health Services Administration. 
Citation: Di Paola A, McCurdy SA, Schick V, Wilkerson JM. Opioid Misuse Among Sexual and Gender Minorities in Texas: The Most Vulnerable Populations. J Addiction Prevention. 2021;9(1): 7.

ISSN: $2330-2178$

2. Seth P, Kariisa M, Wilson N, Baldwin G (2019) Drug and Opioid-Involved Overdose Deaths - United States, 2013-2017. MMWR. Morbidity and mortality weekly report 67: 1419-1427.

3. Wilson N, Kariisa M, Seth P, Smith H, Nicole L (2020) Davis, Drug and opioidinvolved overdose deaths-United States, 2017-2018. MMWR 69: 290-297.

4. Becker WC, Fiellin DA (2020) When Epidemics Collide: Coronavirus Disease 2019 (COVID-19) and the Opioid Crisis. Ann Intern Med 173: 59-60.

5. Slavova S, Rock P, Bush HM, Quesinberry D, Walsh S I (2020) Signal of increased opioid overdose during COVID-19 from emergency medical services data. Drug Alcohol Depend 214: 108176.

6. (CDC), C.f.D.C.a.P (2020) Increase in Fatal Drug Overdoses Across the United States Driven by Synthetic Opioids Before and During the COVID-19 Pandemic, C.H.A. Network, Editor. Centers for Disease Control and Prevention (CDC)

7. U.S. Department of Health and Human Services National Institute of Drug Abuse (NIDA) (2020) Opioid summarize by state.

8. Karacostas C (2017) Scale of Opioid Epidemic in Texas likely Obscured by Bad Data, Experts Say, in The Daily Texan.

9. Han B, Compton WM, Blanco C, Crane E, Lee J, et al. (2017) Prescription opioid use, misuse, and use disorders in US adults: 2015 National Survey on Drug Use and Health. Annals Int Med 167: 293-301.

10. Medley G, Lipari RN, Bose J, RTI International: Cribb DS, et al. (2016) Sexual orientation and estimates of adult substance use and mental health: Results from the 2015 National Survey on Drug Use and Health. Substance Abuse and Mental Health Services Administration.

11. Kerridge BT, Pickering RP, Saha TD, Ruan WJ, Chou SP, et al. (2017) Prevalence, sociodemographic correlates and DSM-5 substance use disorders and other psychiatric disorders among sexual minorities in the United States. Drug Alcohol Depend 170: 82-92.

12. Kecojevic A, Jun HJ, Reisner SL, Corliss HL (2017) Concurrent polysubstance use in a longitudinal study of US youth: associations with sexual orientation. Addiction 112: 614-624.

13. Duncan DT, Zweig S, Hambrick HR, Joseph J Palamar JL (2019) Sexual Orientation Disparities in Prescription Opioid Misuse Among U.S. Adults. Am J Prev Med 56: 17-26.

14. Compton WM, Jones CM, Baldwin GT (2016) Relationship between nonmedical prescription-opioid use and heroin use. NEJM 374: 154-163.

15. Kanouse AB, Compton $P$ (2015) The Epidemic of Prescription Opioid Abuse, the Subsequent Rising Prevalence of Heroin Use, and the Federal Response. J Pain Palliative Care Pharma 29: 102-114.

16. J Cicero T, S Ellis M, Surratt HL, Kurtz SP (2014) The changing face of heroin use in the United States: a retrospective analysis of the past 50 years. JAMA psychiatry $71: 821-826$

17. Lake S, Milloy MJ, Dong H, Hayashi K, Wood E, et al. (2016) Initiation into prescription opioid injection and associated trends in heroin use among people who use illicit drugs. Drug Alcohol depend 169: 73-79.

18. Perlman DC, Jordan AE (2018) The syndemic of opioid misuse, overdose, $\mathrm{HCV}$, and HIV: structural-level causes and interventions. Current HIVIAIDS Rep 1-17.

19. Zibbell JE, Iqbal K, Patel RC, Suryaprasad A, Sanders KJ, et al. (2015) Increases in hepatitis $\mathrm{C}$ virus infection related to injection drug use among persons aged $</=30$ years - Kentucky, Tennessee, Virginia, and West Virginia, 2006-2012. MMWR Morb Mortal Wkly Rep 64: 453-458.

20. Conrad C, Bradley HM, Broz D, Buddha S, Chapman EL, et al. (2015) Community outbreak of HIV infection linked to injection drug use of oxymorphone-Indiana, 2015. MMWR. Morbidity and mortality weekly report 64: 443

21. Seth P, Scholl L, Rudd RA, Bacon S (2018) Overdose deaths involving opioids, cocaine, and psychostimulants-United States, 2015-2016. Morbidity and Mortality Weekly Report 67: 349 -358.
22. Texas Department of State Health Services (2016) Texas HIV surveillance report 2015. HIVISTD Epidemiology and Surveillance Branch: Austin, TX.

23. Center for Disease Control and Prevention (2016) HIV surveillance report 2015. Atlanta, GA

24. Meyer IH (2003) Prejudice, social stress, and mental health in lesbian, gay, and bisexual populations: Conceptual issues and research evidence. Psycho Bull 129: 674-697.

25. Meyer IH (2015) Resilience in the study of minority stress and health of sexual and gender minorities. Psychology of sexual orientation and gender diversity 2: 209-213.

26. Meyer IH (1995) Minority stress and mental health in gay men. J Health Soc Behav 38-56.

27. Bradford J, Reisner SL, Honnold JA, Xavieret J (2013) Experiences of transgender-related discrimination and implications for health: results from the Virginia Transgender Health Initiative Study. Am J Public Health 103: 1820-1829.

28. Russell ST, Joyner K (2001) Adolescent sexual orientation and suicide risk: Evidence from a national study. Am J Public Health 91: 1276-1281.

29. McKirnan DJ, Peterson PL (1988) Stress, expectancies, and vulnerability to substance abuse: a test of a model among homosexual men. J Abnorm Psychol 97: 461

30. Feinstein BA, Dyar C, B. London B (2017) Are Outness and Community involvement risk or protective factors for alcohol and drug abuse among sexual minority women? Arch Sex Behav 46: 1411-1423.

31. Wong CF, Weiss G, Ayala G, Kipke MD (2010) Harassment, discrimination, violence, and illicit drug use among young men who have sex with men. AIDS Educ Prev 22: 286-298

32. Institute of Medicine (2011) The health of lesbian, gay, bisexual, and trangender people: Building a foundation for better understadning. Washington, D.C

33. Valentine SE, Shipherd JC (2018) A systematic review of social stress and mental health among transgender and gender non-conforming people in the United States. Clin Psychol Rev 66: 24-38.

34. Martos AJ, Wilson PA, Meyer IH (2017) Lesbian, gay, bisexual, and transgender (LGBT) health services in the United States: Origins, evolution, and contemporary landscape. PloS one 12: e0180544.

35. Substance Abuse and Mental Health Services Administration (2012) Top health issues for LGBT populations information and resource kit. Rockville.

36. Gonzales G, Henning-Smith C (2017) Health Disparities by Sexual Orientation: Results and Implications from the Behavioral Risk Factor Surveillance System. J Community Health 42: 1163-1172.

37. Grey JA, Konstan J, lantaffi A, Wilkerson JM, Galos D (2015) An updated protocol to detect invalid entries in an online survey of men who have sex with men (MSM): how do valid and invalid submissions compare? AIDS and Behav 19: 1928-1937.

38. Reisner SL, Biello K, Rosenberger JG, Austin SB, Haneuse S (2014) Using a two-step method to measure transgender identity in Latin America/the Caribbean, Portugal, and Spain. Arch sex behav 43: 1503-1514.

39. Yin S, Njai R, Barker L, Siegel PZ, Liao Y, et al. (2016) Summarizing healthrelated quality of life (HRQOL): development and testing of a one-factor model. Population health metrics 14: 22.

40. Bostwick W, Hequembourg A (2014) 'Just a little hint': bisexual-specific microaggressions and their connection to epistemic injustices. Cult Health Sex 16: 488-503.

41. Hayfield N, Clarke V, Halliwell E (2014) Bisexual women's understandings of social marginalisation:'The heterosexuals don't understand us but nor do the lesbians'. Feminism \& Psychology 24: 352-372.

42. Wilkerson JM, Noor SW, Galos DL, Simon Rosser BR (2016) Correlates of a Single-Item Indicator Versus a Multi-Item Scale of Outness About Same-Sex Attraction. Arch Sex Behav 45: 1269-1277. 
Citation: Di Paola A, McCurdy SA, Schick V, Wilkerson JM. Opioid Misuse Among Sexual and Gender Minorities in Texas: The Most Vulnerable Populations. J Addiction Prevention. 2021;9(1): 7.

43. Krieger N, Smith K, Naishadham D, Hartman C, Barbeauet EM, et al. (2005) Experiences of discrimination: Validity and reliability of a self-report measure for population health research on racism and health. So Sci Med 61: 15761596.

44. Blake RL, McKay DA (1986) A single-item measure of social supports as a predictor of morbidity. J Fam Pract 22: 82-84.

45. Anna-Liisa E, Leppänen A, Jahkola A (2003) Validity of a single-item measure of stress symptoms. Scand J Work Environ Health 29: 444-451.

46. Bush K, Kivlahan DR, McDonell MB, Fihn SD, Bradley KA, et al. (1998) The audit alcohol consumption questions (audit-c): An effective brief screening test for problem drinking. Arch Intern Med 158: 1789-1795.

47. Babor TF (2001) AUDIT: The alcohol use disorders identification test guidelines for use in primary care. World Health Organization Department of Mental Health and Substance Dependence: Geneva, Switzerland.

48. Hosmer Jr DW, Lemeshow S, Sturdivant RX (2013) Applied logistic regression pp. 398

49. Hosmer Jr DW, Lemeshow S, May S (2011) Applied survival analysis: regression modeling of time-to-event data pp. 618.

50. StataCorp (2015) Stata Statistical Software: Release 14. StataCorp LP College Station, TX.

51. !!! INVALID CITATION !!! 32, 33.

52. Flanders CE (2015) Bisexual health: A daily diary analysis of stress and anxiety. Basic and Applied Social Psychology 37: 319-335.
53. Petroll AE, Mitchell JW (2015) Health Insurance and Disclosure of Same-Sex Sexual Behaviors Among Gay and Bisexual Men in Same-Sex Relationships. LGBT Health 2: 48-54.

54. Macapagal K, Bhatia R, Greene GJ (2016) Differences in Healthcare Access, Use, and Experiences Within a Community Sample of Racially Diverse Lesbian, Gay, Bisexual, Transgender, and Questioning Emerging Adults. LGBT Health 3: 434-442.

55. Gonzales G, Henning-Smith C (2017) Barriers to Care Among Transgender and Gender Nonconforming Adults. Milbank Q 95: 726-748.

56. Gonzales GR, Dedania, Driscoll R (2019) Health Insurance Coverage and Access to Care Among US-Born and Foreign-Born Sexual Minorities. J Immigr Minor Health 21: 540-548.

57. Gonzales G, Driscoll R, Quinones N (2019) Who Are the Remaining Uninsured Sexual Minority Adults Under the Affordable Care Act? LGBT Health 6: 319-325.

58. Griffin-Tomas M, Cahill S, Kapadia F, Perry N (2019) Halkitis, Access to Health Services Among Young Adult Gay Men in New York City. Am J Mens Health 13: 1557988318818683

59. Schaper E, Padwa H, Urada D, Steven Shoptaw S (2016) Substance use disorder patient privacy and comprehensive care in integrated health care settings. Psychol Serv 13: 105-109.

60. Gumbley SJ (2016) Recovery in the 21st Century: From Shame to Strength J Addict Nurs 27: 143-147.

61. (2018) Pew Research Center Internet \& Technology. Social media use in 2018

\section{Acknowledgement}

Funding provided by the University of Texas Health Science Center at Houston School of Public Health.The authors would like to thank all TUTS participants and the staff at The Montrose Center for supporting this study. 